\title{
openheart Fetuin-A is related to infarct size, left ventricular function and remodelling after acute STEMI
}

\author{
Hans-Josef Feistritzer, ${ }^{1}$ Gert Klug, ${ }^{1}$ Sebastian J Reinstadler, ${ }^{1}$ \\ Marie-Therese Gröber, ${ }^{1}$ Johannes Mair, ${ }^{1}$ Rudolf Kirchmair, ${ }^{1}$ Benjamin Henninger, ${ }^{2}$ \\ Wolfgang-Michael Franz, ${ }^{1}$ Bernhard Metzler ${ }^{1}$
}

To cite: Feistritzer H-J, Klug G, Reinstadler SJ, et al Fetuin-A is related to infarct size, left ventricular function and remodelling after acute STEMI. Open Heart 2015;2: e000244. doi:10.1136/ openhrt-2015-000244

Received 22 January 2015 Revised 30 March 2015 Accepted 3 June 2015
CrossMark

\author{
${ }^{1}$ University Clinic of Internal \\ Medicine III, Cardiology and \\ Angiology, Medical University \\ of Innsbruck, Innsbruck, \\ Austria \\ ${ }^{2}$ University Clinic of \\ Radiology, Medical University \\ of Innsbruck, Innsbruck, \\ Austria
}

Correspondence to Dr Bernhard Metzler; Bernhard.Metzler@uki.at

\section{ABSTRACT}

Objective: To investigate the relationship between plasma fetuin-A, an anti-inflammatory glycoprotein which might be involved in myocardial healing after acute infarction, and infarct size, left ventricular (LV) function and dimensions as well as the occurrence of adverse remodelling at 4 months after acute ST segment elevation myocardial infarction (STEMI).

Methods: In this single-centre prospective, observational study, 89 patients underwent cardiac MR within the first week and 4 months after mechanical reperfusion for first STEMI. Infarct size, LV function and dimensions were assessed at both time points. Fetuin-A levels were determined from blood samples drawn at a median of $49 \mathrm{~h}$ (IQR 30-59 h) after STEMI by an immunofluorescent assay.

Results: Fetuin-A levels (median $568 \mu \mathrm{g} / \mathrm{mL}, \mathrm{IQR}$ $478-763 \mu \mathrm{g} / \mathrm{mL}$ ) were significantly correlated with infarct size and LV ejection fraction at baseline and follow-up (all $p<0.05$ ). Moreover, fetuin-A was related to the increase in the end-diastolic volume index $(r=-0.383, p<0.001)$. According to multivariate logistic regression analysis, fetuin- $A$ concentrations ( $H R=0.17$, $95 \% \mathrm{Cl} 0.03$ to $0.89, \mathrm{p}=0.036$ ) besides the presence of late microvascular obstruction (HR=10.03, $95 \% \mathrm{Cl}$ 0.98 to $102.43, p=0.05$ ) were significantly related to the occurrence of adverse LV remodelling at 4 months. Conclusions: Circulating fetuin-A at day 2 after STEMI is related to acute and chronic infarct size, LV function and dimensions. In addition, it might be useful to identify patients at increased risk for adverse LV remodelling.

\section{INTRODUCTION}

Myocardial ischaemic injury triggers an activation of inflammatory pathways. ${ }^{1}$ Subsequently, left ventricular (LV) function declines, and cardiac remodelling is promoted. $^{2}{ }^{3}$ Adverse LV remodelling is associated with a significant worsening of prognosis after acute myocardial infarction. ${ }^{4}$ Cardiac MR (CMR) is the current gold standard in the in vivo quantification of infarct size, LV function and dimensions. ${ }^{5}$

\section{KEY MESSAGES}

What is already known about this subject?

- Myocardial ischaemia is generally followed by the activation of inflammatory pathways. After myocardial ischaemia, plasma levels of the antiinflammatory glycoprotein fetuin-A decrease, a process which might consequently lead to myocardial calcification and fibrosis. To what extent fetuin-A levels are related to infarct size, left ventricular (LV) function and remodelling after acute ST segment elevation myocardial infarction (STEMI) is largely unknown.

What does this study add?

- We demonstrated that plasma fetuin-A levels, measured two days after acute STEMI, are associated with acute and chronic infarct size as well as LV ejection fraction. Furthermore, fetuin-A independently predicts the development of LV remodelling at 4 months after STEMI.

How might this impact on clinical practice?

- Beyond traditional cardiac biomarkers, fetuin-A could help to identify patients with STEMI at increased risk for adverse LV remodelling, if confirmed in larger studies. Since LV remodelling is a major predictor of morbidity and mortality after STEMI, these patients might benefit from intensified medical therapy.

Fetuin-A, also called the $\alpha_{2}$-HeremansSchmid glycoprotein, has well been described as an anti-inflammatory mediator expressed almost exclusively by the liver. ${ }^{6}$ Serum levels of fetuin-A were shown to decrease during inflammatory processes, probably to facilitate the initiation of the healing process. ${ }^{7}$ Furthermore, it increases the solubility of calcium and phosphorus in serum and prevents ectopic soft tissue calcification. ${ }^{8-10}$ Low levels of fetuin-A are associated with a higher severity of coronary artery disease $(\mathrm{CAD}){ }^{11}$ In patients with $\mathrm{CAD}$, fetuin-A levels were inversely 
correlated with the presence of mitral annular calcification and aortic stenosis. ${ }^{12}$

Myocardial calcification and fibrosis are more severe in fetuin-A knockout mice than in the wild-type mice, which are associated with poorer functional recovery after myocardial ischaemia. ${ }^{13}$ In patients with acute coronary syndrome, fetuin-A plasma levels were significantly lower than in healthy controls. ${ }^{14}{ }^{15}$ After ST segment elevation myocardial infarction (STEMI), the decrease of fetuin-A levels over the first 3 days is directly correlated with the degree of myocardial necrosis. ${ }^{16}$ This underlines that myocardial ischaemia leads to inflammatory processes. ${ }^{17}$

To what extent low fetuin-A levels are associated with myocardial damage, LV function and remodelling after acute STEMI remains largely unknown. We hypothesised that low fetuin-A plasma levels at day 2 after STEMI are related to infarct size, decreased $\mathrm{LV}$ function and increased risk for LV remodelling.

\section{METHODS}

\section{Study population}

From April 2011 to June 2014, 89 patients with STEMI were enrolled in this single-centre prospective, observational study at the coronary care unit of the Medical University of Innsbruck. Inclusion criteria were the diagnosis of first acute STEMI according to the redefined ESC/ACG committee (the Joint European Society of Cardiology/American College of Cardiology Committee) criteria and successful reperfusion by primary percutaneous coronary intervention (PCI). ${ }^{18}$ Exclusion criteria were age below 18 years, severe renal dysfunction (estimated glomerular filtration rate $<30 \mathrm{~mL} / \mathrm{min} / 1.73 \mathrm{~m}^{2}$ ), Killip class $>2$ at presentation and contraindications for CMR. Ischaemia time was defined as the time from symptom onset to the time point of first balloon inflation. Data on patient characteristics were obtained, a detailed medical history was taken and physical examination carried out during the inclusion procedure after primary PCI but before the CMR scan and at the 4-month follow-up.

All participants gave written informed consent before inclusion in the trial.

\section{Biochemical measurements}

For measurement of fetuin-A concentrations, EDTA blood samples were collected at a median of $49 \mathrm{~h}$ (IQR $30-59 \mathrm{~h}$ ) after symptom onset and at $127 \pm 19$ days thereafter. They were immediately centrifuged for $10 \mathrm{~min}$ at $2000 \mathrm{~g}$ and stored at $-80^{\circ} \mathrm{C}$ until analysis. Plasma fetuin-A levels were determined by a commercially available sandwich immunofluorescent assay (DFTA00, R\&D Systems Europe, Ltd, Abingdon, UK) after preparing a 4000-fold sample dilution. The analytical sensitivity of the assay is $1.74 \mathrm{ng} / \mathrm{mL}$. Intra-assay and interassay coefficients of variation are $<8.5 \%$ owing to the manufacturer's data. Creatine kinase (CK) activity, high-sensitivity cardiac troponin $\mathrm{T}(\mathrm{cTnT}), \mathrm{C}$ reactive protein $(\mathrm{CRP})$ and N-terminal pro brain natriuretic peptide (NT-proBNP) levels were measured serially and determined as described previously. ${ }^{19}$ The maximum values of these biochemical markers were defined as the highest in the concentration time course.

\section{CMR protocol}

CMR scans were performed $59 \pm 32 \mathrm{~h}$ after symptom onset and at 4 months thereafter (median 123 days, IQR 120127 days). All scans were performed on a 1.5 Tesla Magnetom Avanto scanner (Siemens, Erlangen, Germany). The CMR protocol has been described in detail previously. ${ }^{20}{ }^{21}$ Briefly, cine CMR images in the short axis (11 slices) were acquired using breath hold, retrospective ECG-triggered trueFISP (true fast imaging with steady-state precession) bright-blood sequences. Images were evaluated by an experienced observer using standard software (Argus, Siemens, Erlangen, Germany). Contouring of LV endocardial and epicardial borders was performed semiautomatically. Papillary muscles were excluded from the myocardial mass and included in the LV volume. End-diastolic volume index (EDVI) and endsystolic volume index (ESVI) were calculated by dividing end-diastolic volume (EDV) and end-systolic volume by the body surface area. The most basal slices were excluded from evaluation in the presence of the LV outflow tract, especially on the end-systolic images. ${ }^{22}$ Increase in EDVI was defined as higher absolute EDVI values at 4 months compared with baseline. Adverse remodelling was defined as an increase in EDV $\geq 20 \%$ between the baseline scan and the 4-month follow-up. ${ }^{23}{ }^{24}$ Ten minutes after a bolus injection of $0.1 \mathrm{mmol} / \mathrm{kg}$ gadolinium-based contrast agent (MultiHance, Bracco, Vienna, Austria) with an infusion pump at $2-3 \mathrm{~mL} / \mathrm{s}$, late gadolinium enhancement (LGE) CMR images were acquired by using an ECG-triggered phase-sensitive inversion-recovery single-shot trueFISP sequence with consecutive short-axis slices as described in detail previously. ${ }^{20}{ }^{25}$ The area of LGE was evaluated quantitatively as reported before. ${ }^{5}$ The presence of late microvascular obstruction (MVO) was defined as a persisting area of hypoenhancement within the hyperenhanced myocardium, as reported before. ${ }^{17}$ Furthermore, infarcts were categorised as transmural if there was a transmural extent in at least two consecutive short-axis slices.

\section{Statistical analysis}

We used SPSS Statistics V.19.0.0 (IBM, Armonk, New York, USA) for statistical analysis. To test for normal distribution, the Kolmogorov-Smirnov test was applied. All variables except fetuin-A, maximum cTnT, maximum CRP, maximum NT-proBNP levels and plasma glucose concentrations at admission and ischaemia time were normally distributed. For statistical testing, they were logtransformed to achieve normal distribution. Results for continuous variables are all expressed as mean $\pm \mathrm{SD}$ or as median with IQR if not normally distributed. To test for 
correlations of continuous variables, Pearson's test was used. The z-score was calculated to test for the difference of two independent correlation coefficients. Differences in continuous variables between groups were assessed by Student $\mathrm{t}$ test and analysis of variance (ANOVA) with Bonferroni post-hoc testing. The paired samples Student t test was applied to determine statistically significant changes of CMR parameters during the 4 -month follow-up. $\chi^{2}$-Test was applied to compare categorical variables between groups. To test for independent correlation between increase in EDVI and fetuin-A levels, a multiple linear regression analysis was performed. All variables listed in table 1 were tested in univariate analysis. Variables showing $\mathrm{p}$ values $<0.1$ in univariate analysis were included into the multiple linear regression model. Furthermore, two models of multivariate binary logistic regression analysis were performed to identify determinants of adverse LV remodelling. In the first model, only biomarkers (fetuin-A, cTnT, NT-proBNP, CRP) were included. Baseline infarct size and late MVO were added to a second model. Moreover, receiver operator characteristics (ROC) analyses for the prediction of $\mathrm{LV}$ remodelling were performed. Two-tailed $\mathrm{p}$ values $<0.05$ were considered to indicate statistical significance.

\section{RESULTS}

\section{Patient characteristics}

All initially enrolled patients completed the 4-month follow-up. Baseline characteristics of the overall study cohort as well as of patients with and without LV remodelling are shown in table 2.

CMR characteristics at baseline and at the 4-month follow-up are summarised in table 3 .

Plasma fetuin-A concentrations at baseline (median $568 \mu \mathrm{g} / \mathrm{mL}$, IQR $478-763 \mu \mathrm{g} / \mathrm{mL}$, range 353-1126 $\mu \mathrm{g} / \mathrm{mL}$ ) were significantly higher compared with 4-month values (median $517 \mu \mathrm{g} / \mathrm{mL}$, IQR $438-572 \mu \mathrm{g} / \mathrm{mL}$, range $306-819 \mu \mathrm{g} / \mathrm{mL} ; \mathrm{p}<0.001)$. A statistically significant correlation was detected between baseline and 4-month fetuin-A concentrations $(r=0.501, \mathrm{p}<0.001)$. No significant difference in baseline and 4-month fetuin-A levels was detected between male and female patients (all $\mathrm{p}>0.150$ ). Fetuin-A did not correlate with age (all $\mathrm{p}>0.065$ ), blood pressure (all $\mathrm{p}>0.430$ ), serum creatinine (all $\mathrm{p}>0.670$ ) and estimated glomerular filtration rate (all $\mathrm{p}>0.370$ ). Fetuin-A was not associated with maximum cTnT (all p $>0.690$ ), maximum CK (all p>0.130), maximum NT-proBNP (all $\mathrm{p}>0.235$ ) and CRP peak levels (all $\mathrm{p}>0.120$ ). Fetuin-A levels did not correlate with ischaemia time (all $\mathrm{p}>0.900$ ). Fetuin-A concentrations did not differ significantly

Table 1 Multivariate analysis of predictors of an increase in EDVI

\begin{tabular}{|c|c|c|c|c|}
\hline & \multicolumn{2}{|c|}{ Univariate } & \multicolumn{2}{|c|}{$\begin{array}{l}\text { Multivariate } \\
\text { Model: } R=0.540, p<0.001\end{array}$} \\
\hline & $\bar{r}$ & p Value & $\bar{\beta}$ & p Value \\
\hline Age, years & 0.056 & 0.605 & - & - \\
\hline Female, n (\%) & 10.036 & 0.740 & - & - \\
\hline Body mass index, $\mathrm{kg} / \mathrm{m}^{2}$ & 0.168 & 0.117 & - & - \\
\hline Family history for AMI, n (\%) & 10.011 & 0.919 & - & - \\
\hline Smoking status, n (\%) & -0.151 & 0.161 & - & - \\
\hline $\mathrm{RR}_{\text {sys }}, \mathrm{mm} \mathrm{Hg}$ & -0.088 & 0.415 & - & - \\
\hline $\mathrm{RR}_{\text {dia }}, \mathrm{mm} \mathrm{Hg}$ & -0.017 & 0.875 & - & - \\
\hline Total cholesterol, mg/dL & -0.022 & 0.847 & - & - \\
\hline Plasma glucose at admission, $\mathrm{mg} / \mathrm{dL}$ & 0.020 & 0.852 & - & - \\
\hline Creatinine, $\mathrm{mg} / \mathrm{dL}$ & 0.038 & 0.729 & - & - \\
\hline eGFR, $\mathrm{mL} / \mathrm{min} / 1.73 \mathrm{~m}^{2}$ & -0.012 & 0.909 & - & - \\
\hline Ischaemia time, $\min$ & -0.048 & 0.657 & - & - \\
\hline Vessel disease, n (\%) & -0.075 & 0.486 & - & - \\
\hline cTnT max, ng/L & 0.369 & $<0.001$ & 0.341 & 0.033 \\
\hline NT-proBNP max, ng/L & 0.204 & 0.061 & -0.030 & 0.787 \\
\hline CRP max, mg/dL & 0.042 & 0.698 & - & - \\
\hline Fetuin-A, mg/L & -0.383 & $<0.001$ & -0.372 & 0.001 \\
\hline LVEF baseline, \% & -0.255 & 0.017 & 0.018 & 0.880 \\
\hline EDVI baseline, $\mathrm{mL} / \mathrm{m}^{2}$ & -0.092 & 0.393 & - & - \\
\hline ESVI baseline, $\mathrm{mL} / \mathrm{m}^{2}$ & 0.102 & 0.346 & - & \\
\hline MMED, g & 0.138 & 0.198 & - & - \\
\hline Infarct size, g & 0.351 & 0.001 & -0.031 & 0.843 \\
\hline Late MVO present, n (\%) & 0.321 & 0.002 & 0.106 & 0.363 \\
\hline Transmurality, n (\%) & 0.282 & 0.008 & 0.030 & 0.797 \\
\hline
\end{tabular}


Table 2 Baseline characteristics and post-PCl treatment of the overall study cohort as well as of patients with and without LV remodelling

\begin{tabular}{|c|c|c|c|c|}
\hline \multirow[b]{2}{*}{ Baseline characteristics } & \multirow{2}{*}{$\begin{array}{l}\text { Overall cohort } \\
n=89\end{array}$} & \multicolumn{2}{|l|}{ Remodelling } & \multirow[b]{2}{*}{ p Value } \\
\hline & & No $(n=75)$ & Yes $(n=14)$ & \\
\hline Age, years & $57 \pm 10$ & $56 \pm 10$ & $61 \pm 10$ & 0.134 \\
\hline Female, n (\%) & $13(15)$ & $10(13)$ & $3(21)$ & 0.423 \\
\hline Body mass index, $\mathrm{kg} / \mathrm{m}^{2}$ & $27 \pm 3$ & $26 \pm 3$ & $27 \pm 3$ & 0.298 \\
\hline Family history for AMI, n (\%) & $29(33)$ & $26(35)$ & $3(21)$ & 0.535 \\
\hline Smoking status, $\mathrm{n}(\%)$ & $46(52)$ & $40(53)$ & $6(43)$ & 0.566 \\
\hline Hypertension, n (\%) & $57(64)$ & $47(63)$ & $10(71)$ & 0.763 \\
\hline $\mathrm{RR}_{\mathrm{sys}}, \mathrm{mm} \mathrm{Hg}$ & $129 \pm 25$ & $129 \pm 24$ & $129 \pm 30$ & 0.956 \\
\hline $\mathrm{RR}_{\mathrm{dia}}, \mathrm{mm} \mathrm{Hg}$ & $80 \pm 14$ & $80 \pm 15$ & $78 \pm 14$ & 0.625 \\
\hline Hyperlipidaemia, n (\%) & $55(62)$ & $48(64)$ & $7(50)$ & 0.376 \\
\hline Total cholesterol, mg/dL & $194 \pm 41$ & $193 \pm 37$ & $197 \pm 58$ & 0.830 \\
\hline Diabetes mellitus, n (\%) & $7(8)$ & $7(9)$ & $0(0)$ & 0.591 \\
\hline Plasma glucose, mg/dL & $129(113-155)$ & $129(112-151)$ & $127(116-161)$ & 0.900 \\
\hline CKD, n (\%) & $5(6)$ & $5(7)$ & $0(0)$ & 0.999 \\
\hline Creatinine, $\mathrm{mg} / \mathrm{dL}$ & $0.94 \pm 0.15$ & $0.94 \pm 0.15$ & $0.91 \pm 0.12$ & 0.459 \\
\hline eGFR, $\mathrm{mL} / \mathrm{min} / 1.73 \mathrm{~m}^{2}$ & $88 \pm 16$ & $88 \pm 16$ & $87 \pm 18$ & 0.956 \\
\hline Ischaemia time, min & 197 (140-404) & 197 (143-390) & 209 (122-483) & 0.799 \\
\hline Anterior STEMI, n (\%) & $36(40)$ & $26(35)$ & $10(71)$ & 0.016 \\
\hline Vessel disease, n (\%) & & & & 0.022 \\
\hline 1 & $49(55)$ & $41(55)$ & $8(57)$ & \\
\hline 2 & $31(35)$ & 29 (39) & $2(14)$ & \\
\hline 3 & $9(10)$ & $5(7)$ & $4(29)$ & \\
\hline CK max, U/L & $2543 \pm 2086$ & $2275 \pm 1922$ & $3974 \pm 2409$ & 0.005 \\
\hline cTnT max, ng/L & 5303 (2308-7868) & 4303 (1774-7096) & 7675 (5386-15 208) & $<0.001$ \\
\hline NT-proBNP max, ng/L & 677 (218-1489) & $675(192-1384)$ & $784(606-3658)$ & 0.070 \\
\hline CRP max, mg/dL & $1.7(0.9-3.6)$ & $1.6(0.9-3.3)$ & $2.8(1.4-5.0)$ & 0.308 \\
\hline Fetuin-A, $\mu \mathrm{g} / \mathrm{mL}$ & $568(478-763)$ & $581(484-811)$ & $518(447-574)$ & 0.001 \\
\hline \multicolumn{5}{|l|}{ Post-PCI treatment } \\
\hline Aspirin, n (\%) & $88(99)$ & $75(100)$ & $13(93)$ & 0.157 \\
\hline Clopidogrel, n (\%) & $10(11)$ & $7(9)$ & $3(21)$ & 0.189 \\
\hline Prasugrel, n (\%) & $74(83)$ & $64(85)$ & $10(71)$ & 0.243 \\
\hline Ticagrelor, n (\%) & $5(6)$ & $4(5)$ & $1(7)$ & 0.584 \\
\hline DAPT, n (\%) & $88(99)$ & $75(100)$ & $13(93)$ & 0.157 \\
\hline$\beta$-Blocker, n (\%) & $78(88)$ & $66(88)$ & $12(86)$ & 0.682 \\
\hline Calcium channel blocker, n (\%) & $3(3)$ & $3(4)$ & $0(0)$ & 0.999 \\
\hline ACE inhibitor, $n(\%)$ & $73(82)$ & $61(81)$ & $12(86)$ & 0.999 \\
\hline AT1-antagonist, $\mathrm{n}(\%)$ & $8(9)$ & $8(11)$ & $0(0)$ & 0.347 \\
\hline Statin, n (\%) & $89(100)$ & $75(100)$ & $14(100)$ & - \\
\hline
\end{tabular}

AMI, acute myocardial infarction; AT1, angiotensin II receptor type 1; CK, creatine kinase; CKD, chronic kidney disease; CRP, C reactive protein; cTnT, cardiac troponin T; DAPT, dual antiplatelet therapy; eGFR, estimated glomerular filtration rate; LV, left ventricular; max, maximum; NT-proBNP, N-terminal pro brain natriuretic peptide; $\mathrm{PCl}$, percutaneous coronary intervention; RR $\mathrm{R}_{\mathrm{dia}}$, diastolic blood pressure; $\mathrm{RR}_{\text {sys }}$, systolic blood pressure; STEMI, ST segment elevation myocardial infarction.

between patients with 1-vessel, 2-vessel and 3-vessel disease (all $\mathrm{p}>0.05$ ).

\section{Correlation of fetuin-A levels with CMR findings}

Linear correlations of baseline fetuin-A concentrations and CMR characteristics are shown in table 4 and figure 1 . Baseline fetuin-A levels did not differ significantly between patients presenting with or without late MVO ( $\mathrm{p}=0.163$ ). No significant difference in fetuin-A concentrations was detected between transmural and non-transmural infarcts ( $\mathrm{p}=0.341)$.

Fetuin-A concentrations measured at 4 months were not related to the 4-month infarct size, LV ejection fraction (LVEF), EDVI, ESVI and myocardial muscle mass (all $\mathrm{p}>0.200$ ).

\section{Fetuin-A and $L V$ remodelling}

Patients with an increase in EDVI between baseline and 4 months showed significantly lower baseline fetuin-A levels compared with patients whose EDVI declined (median $538 \mu \mathrm{g} / \mathrm{mL}$, IQR $449-608 \mu \mathrm{g} / \mathrm{mL}$ vs median $735 \mu \mathrm{g} / \mathrm{mL}$, IQR $553-884 \mu \mathrm{g} / \mathrm{mL} ; \mathrm{p}<0.001)$. Baseline fetuin-A concentrations were significantly related to the increase in EDVI between baseline and 4 months $(\mathrm{r}=-0.383, \mathrm{p}<0.001)$ (figure 2$)$. This association was significant even after correction for covariates (table 1). 
Table 3 CMR characteristics at baseline and at the 4-month follow-up

\begin{tabular}{|c|c|c|c|c|c|c|}
\hline & \multicolumn{3}{|l|}{ Baseline } & \multicolumn{3}{|c|}{ 4-Month follow-up } \\
\hline & \multirow{2}{*}{$\begin{array}{l}\text { Overall cohort } \\
n=89\end{array}$} & \multicolumn{2}{|c|}{ Remodelling } & \multirow{2}{*}{$\begin{array}{l}\text { Overall cohort } \\
n=89\end{array}$} & \multicolumn{2}{|c|}{ Remodelling } \\
\hline & & No $(n=75)$ & Yes $(n=14)$ & & No $(n=75)$ & Yes $(n=14)$ \\
\hline LVEF, \% & $55 \pm 10$ & $56 \pm 10$ & $50 \pm 7 \dagger$ & $59 \pm 10^{*}$ & $60 \pm 9$ & $52 \pm 11 \dagger$ \\
\hline EDVI, $\mathrm{mL} / \mathrm{m}^{2}$ & $73.7 \pm 13.9$ & $74.7 \pm 13.8$ & $68.5 \pm 14.0$ & $78.6 \pm 16.3^{*}$ & $76.7 \pm 14.9$ & $88.9 \pm 20.2 \dagger$ \\
\hline ESVI, mL/m² & $34.2 \pm 11.5$ & $34.1 \pm 11.7$ & $34.6 \pm 10.5$ & $32.7 \pm 12.7$ & $31.1 \pm 11.8$ & $41.5 \pm 14.4 \dagger$ \\
\hline MMED, g & $134 \pm 30$ & $134 \pm 29$ & $134 \pm 38$ & $131 \pm 28$ & $130 \pm 26$ & $135 \pm 37$ \\
\hline Infarct size, g & $25 \pm 18$ & $23 \pm 17$ & $36 \pm 19 \dagger$ & $16 \pm 15^{\star}$ & $15 \pm 13$ & $27 \pm 19 \dagger$ \\
\hline Late MVO present, n (\%) & $52(58)$ & $39(52)$ & $13(93) \dagger$ & - & - & - \\
\hline Transmural infarction, $\mathrm{n}(\%)$ & $70(79)$ & $56(75)$ & $14(100) \dagger$ & - & - & - \\
\hline
\end{tabular}

${ }^{*} p<0.001$ vs overall cohort at baseline.

$\mathrm{tp} \leq 0.05$ vs no remodelling.

CMR, Cardiac MR; EDVI, end-diastolic volume index; ESVI, end-systolic volume index; LVEF, left ventricular ejection fraction; MMED,

myocardial mass at end diastole; MVO, microvascular obstruction.

Differences in the baseline characteristics of patients with $(\mathrm{n}=14)$ and without $(\mathrm{n}=75) \mathrm{LV}$ remodelling are shown in table 2. In the first model of multivariate logistic regression analysis, baseline fetuin-A (HR $=0.23,95 \%$ CI 0.06 to $0.95, \mathrm{p}=0.041$ ) but not maximum cTnT, NT-proBNP and CRP (all $\mathrm{p}>0.110$ ) concentrations were associated with the occurrence of adverse LV remodelling at 4 months. According to a second model also including baseline infarct size and late MVO, fetuin-A concentrations $(\mathrm{HR}=0.17,95 \%$ CI 0.03 to $0.89, \mathrm{p}=0.036)$ besides the presence of late MVO (HR=10.03, 95\% CI 0.98 to $102.43, p=0.05$ ) were significantly related to the occurrence of adverse LV remodelling.

ROC curves indicating the risk for LV remodelling are shown in figures 3 and 4 .

\section{DISCUSSION}

The main findings of the present study are as follows: fetuin-A levels measured 2 days after acute STEMI are associated with acute and chronic infarct size as well as LVEF as assessed by CMR. Moreover, fetuin-A predicts the development of LV remodelling post-STEMI.

Myocardial necrosis is followed by inflammation, ${ }^{1}$ which is a trigger for cardiac remodelling and subsequent development of heart failure. ${ }^{26}$ Low levels of antiinflammatory mediators were related to poor prognosis after acute myocardial infarction. ${ }^{27}$ Fetuin-A is an anti- inflammatory glycoprotein and its plasma concentrations decrease during inflammatory processes. ${ }^{7}$ Fetuin-A levels are lower in patients with STEMI compared with healthy controls and decrease over the first 3 days after symptom onset. ${ }^{15}{ }^{16}$ We showed that plasma fetuin-A concentrations measured 4 months after infarction were significantly lower compared with baseline values. This finding might suggest that plasma fetuin-A concentrations decrease beyond the first few days after the acute event. As shown by Cubedo et $a l,{ }^{16}$ the decline in serum fetuin-A levels early after acute STEMI is correlated with the degree of myocardial necrosis. In this study, baseline but not 4-month fetuin-A concentrations were associated with acute and chronic infarct size. These findings indicate that the decrease of plasma fetuin-A levels within the first few days after infarction as well as single time point fetuin-A concentrations at baseline might be useful to predict infarct size. ${ }^{7}{ }^{13}$ In this study, baseline and 4-month fetuin-A levels did not differ significantly between patients presenting with 1-vessel, 2-vessel and 3-vessel disease. Anyhow, low fetuin-A levels have been shown to be associated with a higher severity of CAD in a community-dwelling population. ${ }^{11}$ We speculate that the impact of stable CAD on fetuin-A levels might be masked following myocardial infarction. This is possibly due to a prolonged inflammatory response within the myocardium, which depresses plasma fetuin-A concentrations.

Table 4 Correlation of BL fetuin-A concentrations with $C M R$ characteristics at $B L$ and 4 months

\begin{tabular}{|c|c|c|c|c|c|c|}
\hline & \multicolumn{2}{|l|}{ BL } & \multicolumn{2}{|c|}{4 Months } & \multicolumn{2}{|c|}{ BL to 4 months } \\
\hline & $r$ & p Value & $r$ & p Value & $r$ & p Value \\
\hline LVEF, \% & 0.252 & 0.017 & 0.334 & 0.001 & 0.103 & 0.338 \\
\hline EDVI, $\mathrm{mL} / \mathrm{m}^{2}$ & -0.124 & 0.247 & -0.336 & 0.001 & -0.383 & $<0.001$ \\
\hline ESVI, $\mathrm{mL} / \mathrm{m}^{2}$ & -0.210 & 0.048 & -0.339 & 0.001 & -0.236 & 0.027 \\
\hline MMED, g & -0.236 & 0.026 & -0.253 & 0.017 & 0.001 & 0.989 \\
\hline Infarct size, g & -0.266 & 0.012 & -0.226 & 0.033 & 0.145 & 0.175 \\
\hline
\end{tabular}

BL, baseline; CMR, cardiac MR; EDVI, end-diastolic volume index; ESVI, end-systolic volume index; LVEF, left ventricular ejection fraction; MMED, myocardial mass at end diastole. 

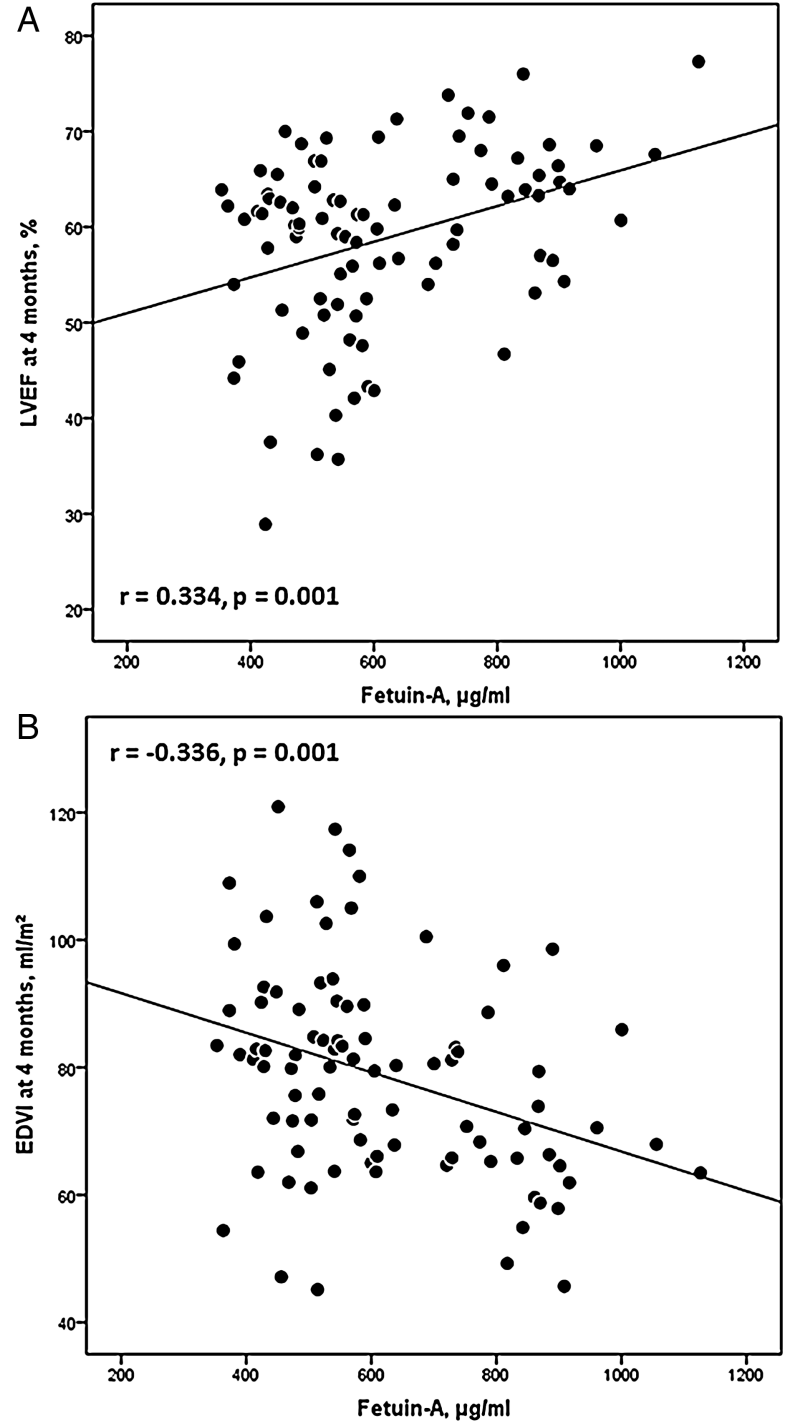

Figure 1 (A) Linear correlation of fetuin-A and left ventricular ejection fraction (LVEF) at 4 months. (B) Linear correlation of fetuin-A and end-diastolic volume index (EDVI) at 4 months.

The decrease in fetuin-A levels is accompanied by the aggravated synthesis of proinflammatory cytokines, which depress ventricular function and promote remodelling. ${ }^{2}$ For the first time, we report in this study a significant correlation between 2-day fetuin-A levels and CMR-derived LVEF in the acute and chronic settings after STEMI. This is in line with data previously published by Lim et $a l^{15}$ who utilised echocardiography and ventriculography for the assessment of LV function, volumes and morphology, which are less-accurate imaging modalities than CMR. ${ }^{28}$

Besides the adverse effect on LV function, inflammatory processes promoting calcification and fibrosis also trigger subsequent dilation of the left ventricle. ${ }^{2}{ }^{3}$ In this study, low fetuin-A levels were associated with 4-month EDVI. Moreover, fetuin-A concentrations were independently associated with the increase in EDVI during follow-up. Fetuin-A was independently related to the occurrence of adverse LV remodelling, even after

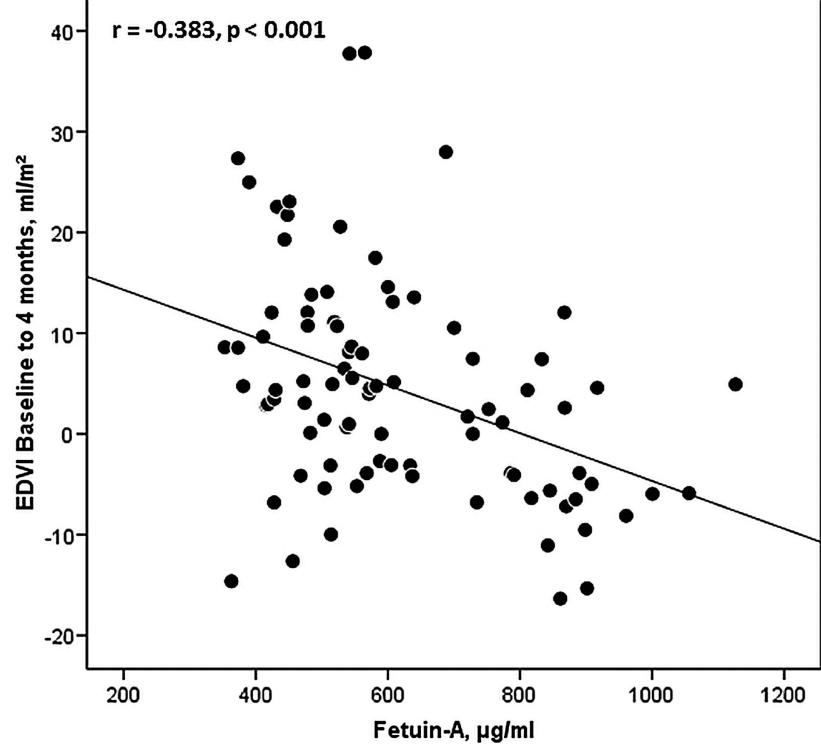

Figure 2 Linear correlation of fetuin-A and the change in end-diastolic volume index (EDVI) between baseline and 4 months.

correction for traditional laboratory and CMR-derived determinants of cardiac remodelling. ${ }^{29}$ According to ROC analyses, adding fetuin-A to a model including maximum cTnT, NT-proBNP and CRP concentrations resulted in a higher area under the curve for the prediction of adverse LV remodelling. These findings are in

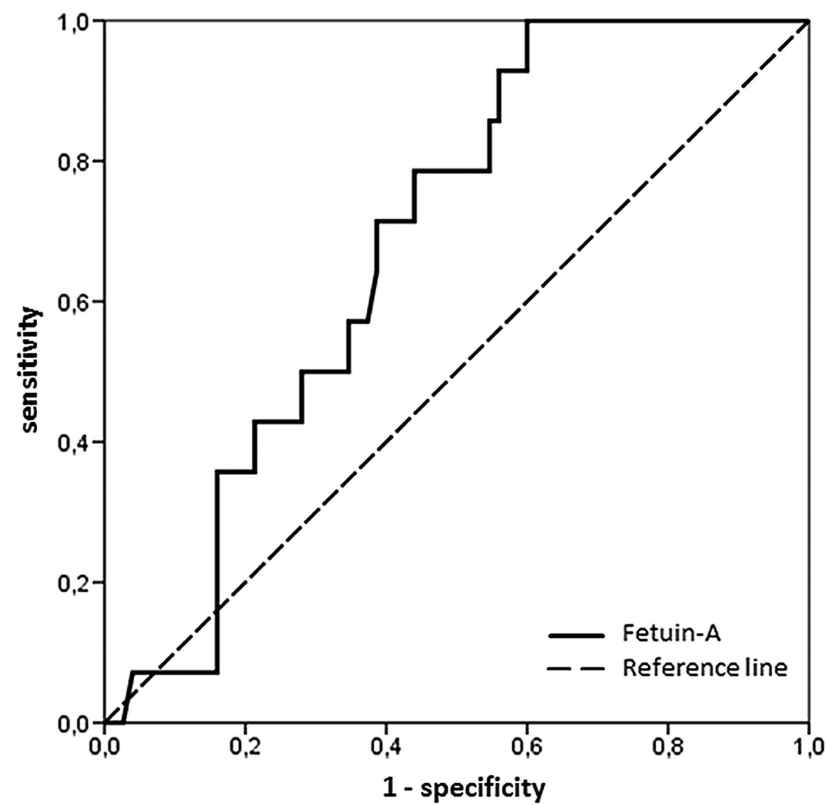

Figure 3 Analysis of receiver operating characteristic of fetuin-A. The area under the curve of fetuin- $\mathrm{A}(0.68,95 \% \mathrm{Cl}$ 0.56 to 0.80 ) with the optimal cut-off value of $544 \mu \mathrm{g} / \mathrm{mL}$ provided $71 \%$ sensitivity and $61 \%$ specificity for the prediction of adverse left ventricular remodelling at 4 months. The corresponding negative and positive predictive values were $92 \%$ and $26 \%$. 


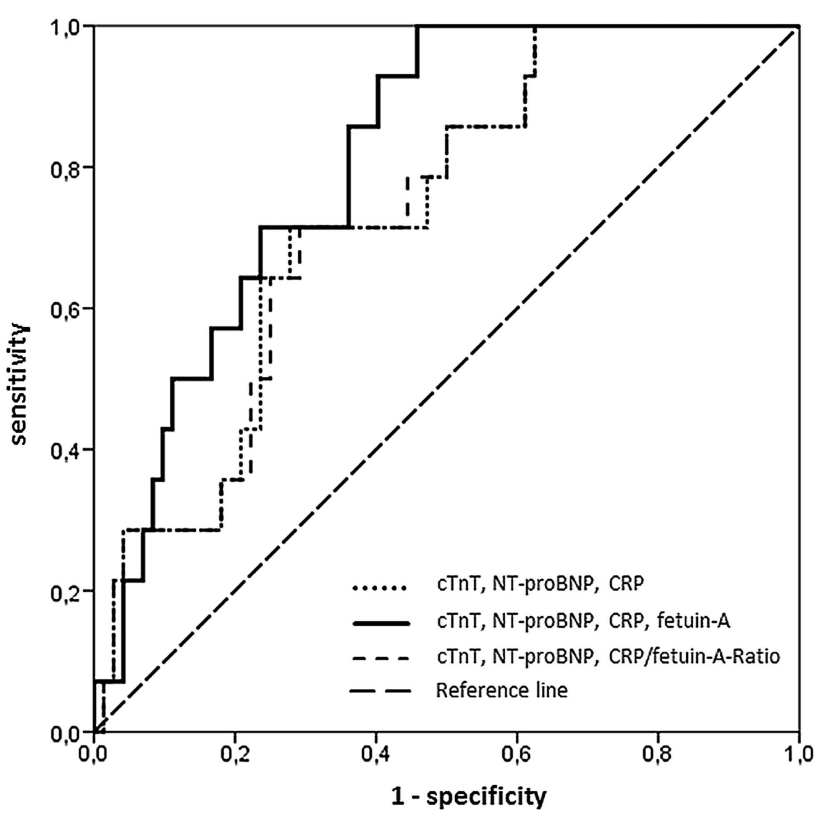

Figure 4 Analysis of receiver operating characteristic of three models (model 1: cardiac troponin T (cTnT), $\mathrm{N}$-terminal pro brain natriuretic peptide (NT-proBNP), $\mathrm{C}$ reactive protein (CRP) (dotted line); model 2: cTnT, NT-proBNP, CRP and fetuin-A (continuous line); model 3: cTnT, NT-proBNP and CRP/fetuin-A-ratio (broken line)) for prediction of adverse left ventricular remodelling at 4 months. Model 2 (area under the curve $=0.812,95 \% \mathrm{Cl} 0.712$ to 0.911 ) resulted in a higher prognostic value for the prediction of adverse remodelling compared with model 1 (area under the curve $=0.736,95 \% \mathrm{Cl}$ 0.609 to 0.863 ) and model 3 (area under the curve $=0.735$, $95 \% \mathrm{Cl} 0.609$ to 0.861 ).

agreement with those of Merx $e t a l^{13}$ who reported that low levels of fetuin-A negatively affect cardiac structure and function in a murine model of myocardial ischaemia. Nevertheless, larger studies with a longer period of follow-up should be performed to confirm our findings.

\section{Study limitations}

This study has some important limitations. Although patients with STEMI were screened consecutively, only haemodynamically stable patients with a first coronary event and preserved renal function were included. These facts resulted in a homogeneous study cohort with a moderate infarct size and a relatively preserved LVEF. Anyhow, the baseline characteristics of our study population are comparable to large multicentre trials (median LVEF $51 \%$ ), which also exhibited a similar ischaemia time. ${ }^{30}$ Second, in this study, only single time point fetuin-A levels were measured at baseline. Therefore, the optimal time point for fetuin-A measurement with the highest prognostic value for the prediction of $\mathrm{LV}$ remodelling remains unknown. Future studies performing serial measurements of fetuin-A over the first 2 days after symptom onset are needed to determine the optimal time point for measurement of this biomarker. Moreover, the remodelling group was too small to draw robust conclusions. Accordingly, our findings should be confirmed in larger studies. Although higher fetuin-A plasma levels have been described in females compared with males, ${ }^{31}$ this study was not powered to point out any gender-related differences. Another limitation is that data on staged revascularisation of the non-infarct-related artery could not be assessed for all patients, primarily due to non-in-house procedures.

\section{CONCLUSION}

The anti-inflammatory glycoprotein fetuin-A might be involved in myocardial healing after acute myocardial infarction. For the first time, this study links 2-day fetuin-A concentrations to CMR-derived infarct size, LV function and morphology in the acute and chronic stage after STEMI. Moreover, fetuin-A might be of additional prognostic value in the prediction of adverse LV remodelling, which should be confirmed in larger studies.

Acknowledgements The authors thank Ursula Stanzl for valuable technical assistance.

Contributors H-JF contributed in the analysis and interpretation of the data; and in the idea, design and drafting of the manuscript. GK contributed in the analysis and interpretation of the data; and in the design, drafting and critical revision of the manuscript for important intellectual content. SJR contributed in the design, drafting and critical revision of the manuscript for important intellectual content. M-TG and RK contributed in the analysis of the data; and in the critical revision of the manuscript for important intellectual content. JM and W-MF contributed in the interpretation of the data; and in the critical revision of the manuscript for important intellectual content. BH contributed in the analysis and interpretation of the data; and in the critical revision of the manuscript for important intellectual content. W-MF contributed in the interpretation of the data; and in the critical revision of the manuscript for important intellectual content. BM contributed in the interpretation of the data; and in the design and critical revision of the manuscript for important intellectual content; and also gave final approval of the manuscript submitted.

Funding This study was supported by grants from the Austrian Society of Cardiology to GK, SJR and BM and by the intramural funding programme of the Medical University of Innsbruck for young scientists, MUI-START, project 2013042016 to GK.

Competing interests None declared.

Patient consent Obtained.

Ethics approval Local ethics committee of the Medical University of Innsbruck.

Provenance and peer review Not commissioned; externally peer reviewed.

Data sharing statement No additional data are available.

Open Access This is an Open Access article distributed in accordance with the Creative Commons Attribution Non Commercial (CC BY-NC 4.0) license, which permits others to distribute, remix, adapt, build upon this work noncommercially, and license their derivative works on different terms, provided the original work is properly cited and the use is non-commercial. See: http:// creativecommons.org/licenses/by-nc/4.0/

\section{REFERENCES}

1. Abbate A, Bonanno E, Mauriello A, et al. Widespread myocardial inflammation and infarct-related artery patency. Circulation 2004;110:46-50.

2. Kelly RA, Smith TW. Cytokines and cardiac contractile function. Circulation 1997;95:778-81.

3. Krown KA, Yasui K, Brooker MJ, et al. TNF alpha receptor expression in rat cardiac myocytes: TNF alpha inhibition of L-type Ca2+ current and Ca2+ transients. FEBS Lett 1995;376:24-30. 
4. Cohn JN, Ferrari R, Sharpe N. Cardiac remodeling-concepts and clinical implications: a consensus paper from an international forum on cardiac remodeling. Behalf of an International Forum on Cardiac Remodeling. J Am Coll Cardiol 2000;35:569-82.

5. Klug G, Metzler B. Assessing myocardial recovery following ST-segment elevation myocardial infarction: short- and long-term perspectives using cardiovascular magnetic resonance. Expert Rev Cardiovasc Ther 2013;11:203-19.

6. Ombrellino $\mathrm{M}$, Wang $\mathrm{H}$, Yang $\mathrm{H}$, et al. Fetuin, a negative acute phase protein, attenuates TNF synthesis and the innate inflammatory response to carrageenan. Shock 2001;15:181-5.

7. Lebreton JP, Joisel F, Raoult JP, et al. Serum concentration of human alpha $2 \mathrm{HS}$ glycoprotein during the inflammatory process: evidence that alpha 2 HS glycoprotein is a negative acute-phase reactant. J Clin Invest 1979;64:1118-29.

8. Schafer C, Heiss A, Schwarz A, et al. The serum protein alpha 2-Heremans-Schmid glycoprotein/fetuin-A is a systemically acting inhibitor of ectopic calcification. J Clin Invest 2003;112:357-66.

9. Heiss A, DuChesne A, Denecke B, et al. Structural basis of calcification inhibition by alpha 2-HS glycoprotein/fetuin-A. Formation of colloidal calciprotein particles. J Biol Chem 2003;278:13333-41.

10. Price PA, Lim JE. The inhibition of calcium phosphate precipitation by fetuin is accompanied by the formation of a fetuin-mineral complex. J Biol Chem 2003;278:22144-52.

11. Ix JH, Barrett-Connor E, Wassel CL, et al. The associations of fetuin-A with subclinical cardiovascular disease in community-dwelling persons: the Rancho Bernardo Study. J Am Coll Cardiol 2011;58:2372-9.

12. Ix JH, Chertow GM, Shlipak MG, et al. Association of fetuin-A with mitral annular calcification and aortic stenosis among persons with coronary heart disease: data from the Heart and Soul Study. Circulation 2007;115:2533-9.

13. Merx MW, Schafer C, Westenfeld R, et al. Myocardial stiffness, cardiac remodeling, and diastolic dysfunction in calcification-prone fetuin-A-deficient mice. J Am Soc Nephrol 2005;16:3357-64.

14. Afsar CU, Uzun $H$, Yurdakul S, et al. Association of serum fetuin-A levels with heart valve calcification and other biomarkers of inflammation among persons with acute coronary syndrome. Clin Invest Med 2012;35:E206-15.

15. Lim P, Collet JP, Moutereau S, et al. Fetuin-A is an independent predictor of death after ST-elevation myocardial infarction. Clin Chem 2007:53:1835-40.

16. Cubedo J, Padro T, Badimon L. Coordinated proteomic signature changes in immune response and complement proteins in acute myocardial infarction: the implication of serum amyloid P-component. Int J Cardiol 2013;168:5196-204.

17. Mayr A, Klug G, Schocke M, et al. Late microvascular obstruction after acute myocardial infarction: relation with cardiac and inflammatory markers. Int J Cardiol 2012;157:391-6.

18. Alpert JS, Thygesen K, Antman E, et al. Myocardial infarction redefined-a consensus document of The Joint European Society of
Cardiology/American College of Cardiology Committee for the redefinition of myocardial infarction. J Am Coll Cardio 2000;36:959-69.

19. Reinstadler S, Klug G, Feistritzer HJ, et al. Relation of plasma adiponectin levels and aortic stiffness after acute ST-segment elevation myocardial infarction. Eur Heart J Acute Cardiovasc Care 2014;3:10-17.

20. Klug G, Trieb T, Schocke M, et al. Quantification of regional functional improvement of infarcted myocardium after primary PTCA by contrast-enhanced magnetic resonance imaging. J Magn Reson Imaging 2009;29:298-304.

21. Feistritzer HJ, Reinstadler SJ, Klug G, et al. Comparison of an oscillometric method with cardiac magnetic resonance for the analysis of aortic pulse wave velocity. PLOS ONE 2015;10: e0116862.

22. Beek AM, Kuhl HP, Bondarenko O, et al. Delayed contrastenhanced magnetic resonance imaging for the prediction of regional functional improvement after acute myocardial infarction. J Am Coll Cardiol 2003;42:895-901.

23. Bolognese L, Neskovic AN, Parodi G, et al. Left ventricular remodeling after primary coronary angioplasty: patterns of left ventricular dilation and long-term prognostic implications. Circulation 2002;106:2351-7.

24. Reinstadler SJ, Klug G, Feistritzer HJ, et al. Association of copeptin with myocardial infarct size and myocardial function after ST segment elevation myocardial infarction. Heart 2013;99:1525-9.

25. Klug G, Feistritzer HJ, Reinstadler SJ, et al. Association of aortic stiffness with biomarkers of myocardial wall stress after myocardial infarction. Int J Cardiol 2014;173:253-8.

26. Nian M, Lee $\mathrm{P}$, Khaper $\mathrm{N}$, et al. Inflammatory cytokines and postmyocardial infarction remodeling. Circ Res 2004;94:1543-53.

27. Chalikias GK, Tziakas DN, Kaski JC, et al. Interleukin-18: interleukin-10 ratio and in-hospital adverse events in patients with acute coronary syndrome. Atherosclerosis 2005;182:135-43.

28. Bellenger NG, Davies LC, Francis JM, et al. Reduction in sample size for studies of remodeling in heart failure by the use of cardiovascular magnetic resonance. J Cardiovasc Magn Reson 2000;2:271-8.

29. van Kranenburg $\mathrm{M}$, Magro $\mathrm{M}$, Thiele $\mathrm{H}$, et al. Prognostic value of microvascular obstruction and infarct size, as measured by CMR in STEMI patients. JACC Cardiovasc Imaging 2014;7:930-9.

30. Eitel I, Wohrle J, Suenkel H, et al. Intracoronary compared with intravenous bolus abciximab application during primary percutaneous coronary intervention in ST-segment elevation myocardial infarction: cardiac magnetic resonance substudy of the AIDA STEMI trial. J Am Coll Cardiol 2013;61:1447-54.

31. Laughlin GA, Cummins KM, Wassel CL, et al. The association of fetuin-A with cardiovascular disease mortality in older communitydwelling adults: the Rancho Bernardo study. J Am Coll Cardiol 2012;59:1688-96. 